\title{
AVALIAÇÃO DO DESENVOLVIMENTO DA MOTRICIDADE GLOBAL EM CRIANÇAS
}

Deborah Cristina Gonçalves Luiz Fernani ${ }^{1}$, Gabriela Santa Maria Lucin ${ }^{2}$, Laís Bezerra Escarelli ${ }^{2}$, Graziela Cristina Claudino Gomes ${ }^{2}$, Natália Silva Loosli ${ }^{2}$, Renata Aparecida de Oliveira Lima ${ }^{1}$, Francis Lopes Pacagnelli ${ }^{1}$

${ }^{1}$ Docente $\mathrm{e}^{2}$ discentes do Curso de Fisioterapia da UNOESTE, Presidente Prudente, SP.

\section{RESUMO}

O desenvolvimento é a contínua alteração no comportamento global ao longo do ciclo da vida do ser humano, devido à interação entre as necessidades da tarefa, da genética do indivíduo e das condições do ambiente no qual se encontra. A fim de avaliar possíveis alterações ao longo do desenvolvimento de crianças, existem vários testes que podem ser realizados, entre eles o Teste de Denver II composto por quatro áreas: pessoal-social, motor fino adaptativo, linguagem e motor-global, sendo este último o instrumento deste estudo. Apresentou-se como objetivo avaliar a motricidade global em de crianças de creche. Participaram 17 crianças entre 10 e 30 meses de idade cronológica, média de 22,7 meses, sendo 9 (52,95\%) do sexo masculino e $8(47,05 \%)$ do sexo feminino, das 21 frequentadoras de um berçário inserido em uma creche na periferia de Presidente Prudente, SP. Todas apresentaram seus resultados em "aprovado", ou seja, permitiram e realizaram os testes adequados a sua idade cronológica. Posteriormente, classificando a área da motricidade global segundo o Teste de Denver II, 14 crianças (82,36\%) obtiveram o score "avançado" e 3 crianças $(17,64 \%)$ o score "normal". Quanto ao gênero, o desempenho foi ligeiramente melhor nas meninas, pois todas as crianças do sexo feminino apresentaram-se além à idade cronológica. Portanto, o desenvolvimento motor global de crianças de creche pelos métodos utilizados neste estudo apresenta-se "normal" e "avançado" de acordo com a idade cronológica.

Palavras-chave: Desenvolvimento infantil, habilidade, criança, avaliação de desempenho.

\section{EVALUATION OF OVERALL MOTOR DEVELOPMENT IN CHILDREN FROM KINDERGARTEN}

\begin{abstract}
Development is the continuous change in global behavior over the life cycle of human beings, due to the interaction between the needs of the task, the individual's genetic and environmental conditions in which the child is. In order to evaluate possible changes during the development of children, several tests may be performed, including the Denver II Test composed of four assessment items: personal-social, fine motor adaptation, language and overall motor skills, being the latter the instrument used in this study. The objective was to evaluate the overall motor skills of children from a day care center. Seventeen children between 10 and 30 months of chronological age, average of 22.7 months old, participated, being $9(52.95 \%)$ males and 8 (47.05\%) females, of 21 children who join the nursery at a day care center in Presidente Prudente, SP. All children presented tests as "approved", in other words, they performed the activities suitable for the chronological age. Classifying the global motor according to the Development Screening Test (Denver II): 14 children (82.36\%) achieved the score "advanced" and 3 children (17.64\%) were classified as "normal". Regarding gender, the performance was slightly better in girls, because all the female children are beyond the item corresponding to the chronological age. Thus, the overall motor development in children from day care center evaluated by the methods used in this study was "normal" and "advanced" according to the chronological age.
\end{abstract}

Keywords: Child development, skill, child, performance evaluation. 


\section{INTRODUÇÃO}

O desenvolvimento infantil é um processo que começa desde a vida intrauterina, abrangendo vários aspectos, como crescimento físico, maturação neurológica e construção de habilidades vinculadas ao comportamento, às esferas cognitivas, social e afetiva, tornando a criança capaz de responder às suas necessidades e às do seu meio, de acordo com o seu tempo de vida (De SOUZA \& MELLO, 2004; MIRANDA et al., 2003).

A evolução estáticomotora do neonato até a idade adulta depende da maturação do sistema nervoso central, sendo determinada por padrões geneticamente estabelecidos e estímulos ambientais, os quais são captados pelos órgãos do sentido e respondidos pelo cérebro como órgão de integração e coordenação, com reações complexas que decorrem automaticamente (FLEHMING, 2005).

Cada criança apresenta seu padrão característico, mas deve exibir manifestações de desenvolvimento características da idade em que ela se encontra. Com sua evolução, há uma constante observação no crescimento das estruturas somáticas e aumento das possibilidades individuais de agir sobre 0 ambiente (RAFAEL et al., 2010)

O desenvolvimento motor de uma criança é basicamente dividido em duas etapas: habilidade motora global e fina. A habilidade motora global é classicamente definida como a que envolve em sua manifestação a mobilização de grandes grupos musculares produtores de força do tronco, braços e pernas e inclui as reações posturais, o equilíbrio da cabeça, o sentar, o ficar em pé, o engatinhar e o andar (WILLRICH et al., 2006). A habilidade motora fina só se desenvolve depois de a criança ter dominado os movimentos ligados aos grandes músculos. A motricidade fina, como abotoar camisas e desenhar figuras, envolve a coordenação de músculos pequenos e coordenação entre olhos e mãos. Estas habilidades permitem às crianças maior senso de responsabilidade e cuidado pessoal (HABECHIAN \& SANTOS, 2008).

A observação da motricidade global e fina da criança permite detectar como o desenvolvimento está se apresentando, facilitando a promoção de uma internação precoce para os desvios supostamente encontrados. Essa observação pode ser feita por testes. Entre eles, o Teste de Denver II, que é destinado a crianças com a faixa etária de 0 a 6 anos de idade que estão saudáveis, sem nenhuma doença, não sendo designado para gerar diagnósticos ou prever adaptações futuras e capacidades intelectuais, sendo melhor usado para comparar um dado desempenho da criança em uma variedade de tarefas com o desempenho de outras crianças da mesma idade (TECKLIN, 2003).

A creche vem se tornando uma necessidade significativa da população, em consequência das transformações socioeconômicas que a sociedade está sofrendo. Por esse motivo, é importante avaliar o desempenho motor em crianças desse local, pois é neste ambiente que passam a maior parte do dia, sendo um cenário contribuinte para o desenvolvimento de suas habilidades motoras (BISCEGLI et al., 2006).

O objetivo desse estudo foi avaliar o desenvolvimento da motricidade global em crianças de creche.

\section{MÉTODOS}

O estudo foi realizado com crianças de uma creche inserida em escola municipal localizada na periferia da cidade de Presidente Prudente, SP, a qual apresenta, em média, 60 crianças frequentadoras. $\mathrm{O}$ projeto (protocolo 
381/10) foi avaliado e aprovado pelo Comitê de Ética em Pesquisa da UNOESTE.

Foram avaliadas 17 crianças de ambos os sexos, saudáveis, com idade cronológica de 10 a 30 meses, de um total de 21 frequentadoras do berçário. Ter algum problema de saúde ou ser portadora de alguma doença forma critérios de exclusão.

As avaliações ocorreram na própria sala de convivência da criança, com supervisão da cuidadora responsável, sendo baseadas na observação da mesma de acordo com os itens da área "motor-global" do Teste de Denver II, assinalando cada um destes em aprovado (A) quando a criança realiza a ação, falho (F) se a criança não consegue realizar a ação, não oportuno/aplicável (NO) quando a ação não pode ser realizada pela criança ou recusado (R) quando a ação é recusada pela criança (TECKLIN, 2003).

Posteriormente, as crianças foram classificadas em "avançado" (acima do normal), "normal" (nenhum atraso ou, no máximo, um cuidado), "cauteloso" (todo o teste apresentando um atraso ou, pelo menos, dois cuidados), "atrasado" (todo o teste apresentando pelo menos dois atrasos) ou "não oportuno/aplicável" (quando recusas são classificadas como fracassos) (TECKLIN, 2003).

Os resultados das avaliações das crianças foram apresentados aos pais e também à cuidadora responsável pelos mesmos na creche.

A análise dos resultados foi realizada usando estatística descritiva, baseada na freqüência simples e apresentando dados proporcionais.

\section{RESULTADOS}

Foram avaliadas 17 crianças entre 10 e 30 meses de idade cronológica, com média de
22,7 meses, sendo $9(52,95 \%)$ do sexo masculino e $8(47,05 \%)$ do sexo feminino (Tabela 1$)$.

Tabela1. Distribuição das crianças segundo a faixa etária e o gênero.

\begin{tabular}{lcc}
\hline $\begin{array}{l}\text { Idade } \\
\text { (meses) }\end{array}$ & $\begin{array}{c}\text { Feminino } \\
\mathrm{n}(\%)\end{array}$ & $\begin{array}{c}\text { Masculino } \\
\mathrm{n}(\%)\end{array}$ \\
\hline $10-15$ & $1(12,5)$ & $3(33,3)$ \\
$16-20$ & $2(25)$ & $1(11,1)$ \\
$21-25$ & $0(0)$ & $2(22,2)$ \\
$26-30$ & $5(62,5)$ & $3(33,3)$ \\
\hline
\end{tabular}

Com relação à avaliação da motricidade global segundo o Teste de Denver II, todas as crianças apresentaram os testes avaliados como "aprovado" (A), ou seja, realizaram as atividades adequadas para a idade cronológica. Não houve as demais situações: falho $(F)$, não oportuno/aplicável (NO) e/ou recusado (R).

A classificação da motricidade global do Teste de Denver II pode ser observada na Tabela 2.

Tabela 2. Distribuição da avaliação da motricidade global do Teste de Denver II das crianças segundo o gênero.

\begin{tabular}{lcc}
\hline Gênero & $\begin{array}{c}\text { Normal } \\
\mathrm{n}(\%)\end{array}$ & $\begin{array}{c}\text { Avançado } \\
\mathrm{n}(\%)\end{array}$ \\
\hline Feminino & $0(0)$ & $8(47,05)$ \\
Masculino & $3(17,65)$ & $6(35,30)$ \\
Total & $3(17,65)$ & $14(82,35)$ \\
\hline
\end{tabular}

\section{DISCUSSÃO}

Foram avaliadas 17 crianças entre 10 e 30 meses de idade cronológica, com média de 22,7 meses, sendo $9(52,95 \%)$ do sexo masculino e $8(47,05 \%)$ do sexo feminino. Com relação à avaliação da área "motor global" segundo o Teste de Denver II, todas as crianças apresentaram os testes avaliados como "aprovado", ou seja, realizaram as atividades adequadas para a idade cronológica. Não houve as demais situações: falho, não oportuno/aplicável e/ou recusado. De acordo com a classificação da motricidade global do Teste de Denver, 47,05\% obtiveram a 
classificação "avançado" e 17,64\% situaram-se no score "normal".

O Teste de Denver II é um teste de triagem muito utilizado para o acompanhamento do desenvolvimento em crianças normais, mas é também freqüentemente usado para triagem de desvios no desenvolvimento, podendo acelerar o início da intervenção precoce e facilitar 0 desenvolvimento futuro dessas crianças. É considerado de fácil aplicação e rápida execução (SANTOS et al., 2008) característica também observada neste estudo.

Inicialmente, acreditava-se que as mudanças no comportamento motor refletiam diretamente as alterações maturacionais do sistema nervoso central. Hoje, sabe-se que o processo de desenvolvimento ocorre de maneira dinâmica e é suscetível a ser moldado a partir de inúmeros estímulos externos. A interação entre aspectos relativos ao indivíduo, como suas características físicas e estruturais, ao ambiente em que está inserido e à tarefa a ser aprendida são determinantes na aquisição e refinamento das diferentes habilidades (WILLRICH et al., 2006). As creches podem ser ambientes facilitadores deste processo, o que também foi constatado neste trabalho, considerandos 0 desenvolvimento global das mesmas.

Diversos fatores podem colocar em risco o desenvolvimento normal de uma criança. São definidos como fatores de risco uma série de condições biológicas ou ambientais que aumentam a probabilidade de déficits no desenvolvimento neuropsicomotor da criança. Dentre as principais causas de atraso motor encontram-se: baixo peso ao nascer, distúrbios cardiovasculares, respiratórios e neurológicos, infecções neonatais, baixas condições sócio-econômicas, nível educacional precário dos pais ou prematuridade. Quanto maior o número de fatores de risco atuantes, maior será a possibilidade de alteração no desenvolvimento. A amostra deste estudo somente apresenta baixas condições socioeconômicas e nível educacional precário dos pais, no perfil descrito pela direção da creche desses freqüentadores, o que não influenciou na avaliação segundo Teste de Denver II (WILLRICH et al., 2006).

Outro fator que pode estar associado ao atraso no desenvolvimento infantil é a desnutrição. Mesmo crianças que não apresentam sequelas graves podem apresentar comprometimento em algumas áreas do seu desenvolvimento neuropsicomotor, o que prejudica o funcionamento intelectual (BISCEGLI et al., 2007). Uma pesquisa sobre o efeito da desnutrição leve sobre 0 desenvolvimento neuropsicomotor de lactentes confirmou uma tendência de prejuízo nas áreas da linguagem e sociabilidade (MANSUR \& NETO, 2006). Além disso, crianças freqüentadoras de creche recebem praticamente $70 \%$ de suas necessidades nutricionais durante o período de permanência nessas instituições e, por receberem uma alimentação balanceada durante praticamente dez horas do dia, deveriam estar mais protegidas da desnutrição, quando comparadas às crianças da população em geral (BISCEGLI et al., 2007). Não há registro de desnutrição nas crianças da amostra e conseqüência não há atrasos ligados a esta no perfil descrito pela direção do local, que cita que as mesmas sempre são avaliadas por profissionais da saúde.

Nos primeiros anos de vida (12 a 18 meses) existe maior plasticidade cerebral, o que possibilita a otimização de ganhos no desenvolvimento. Nessa perspectiva, diversas pesquisas demonstraram haver melhora da aquisição de habilidades motoras em crianças que receberam estimulação precoce (BARROS et al., 2003; BLAUW \& HADDERS, 2005). A aprendizagem motora é compreendida como uma mudança relativamente permanente de uma 
habilidade motora associada à prática e experiência. Por outro lado, Pilz e Schermann (2007) sugerem que não é necessário ensinar habilidades motoras básicas como agarrar, engatinhar e andar aos bebês. À medida que o sistema nervoso central e músculoesquelético estiverem suficientemente maduros e o ambiente oferecer oportunidades adequadas, os bebês se movimentam e descobrem o que podem fazer. As habilidades surgem à medida que fazem e conseguem explorar. E, quanto mais exploram, mais podem aprender a fazer. Nessa linha de pensamento, vários pesquisadores sugerem que para haver aprendizagem de novas habilidades motoras é necessário dar condições de autoorganização ao bebê através de ambiente e estímulos sensório-motores adequados. O desenvolvimento é interativo e depende da oferta de tarefas ao bebê. A motivação, percepção intrínseca do bebê, características físicas e o ambiente oferecem oportunidades e/ou limitações frente uma meta, objetivos ou tarefa motora. Uma resposta motora emerge como resultado da experimentação de várias tentativas. Então, frente a uma tarefa motora, o indivíduo necessita da possibilidade de prática e experiência, num ambiente adequado, para a execução com sucesso (ANDRADE et al., 2004; SCHOBERT, 2008).

Quanto ao gênero, o desempenho foi ligeiramente melhor nas meninas, pois todas as crianças do sexo feminino estão além do item correspondente à idade cronológica, o que não se observa nas crianças do sexo masculino, onde três delas apresentam-se no item correspondente a sua idade cronológica, também detectado no estudo de Souza et al. (2008), onde as meninas tiveram desempenho melhor que os meninos. Porém, Biscegli et al. (2007), relataram que, com relação ao sexo das crianças, não houve diferença significante quanto ao resultado global do teste ( $37 \%$ suspeitos no feminino e $40 \%$ no masculino). Cabe ressaltar que foram encontrados poucos estudos brasileiros de triagem do desenvolvimento de crianças usando o Teste de Denver II. Tais estudos são, em geral, transversais, em diferentes faixas etárias, realizados em locais diversos (creches públicas e privadas, domicílio e ambulatórios) dificultando a comparação dos resultados.

Há diversos estudos nos quais 0 desempenho motor foi satisfatório, concordante com o obtido por alguns autores que avaliaram crianças de países subdesenvolvidos e verificaram que, mesmo nas classes menos favorecidas, o desenvolvimento motor delas não era afetado, sendo até muitas vezes mais precoce do que 0 de crianças de países desenvolvidos, talvez impulsionados pela necessidade de sobrevivência (SOUZA et al., 2008).

Portanto, o tempo de permanência durante o dia na creche, não só contribui com uma boa alimentação, como também, proporciona a estimulação motora geral dessas crianças, por estarem em um ambiente decorativo, em contato com outras crianças, realizando diferentes atividades, como brincadeiras, jogos, e vivenciando situações do dia-a-dia.

Com o presente estudo pode-se concluir que a motricidade global de crianças de creche apresenta-se "normal" e "avançado" de acordo com a idade cronológica e o Teste de Denver II. Este pode ser utilizado como instrumento de avaliação em instituições que atendam crianças, com o intuito de observar o desenvolvimento destas e, caso este se encontre alterado, iniciar intervenção precoce.

\section{REFERÊNCIAS}

Andrade A, Luft CB, Rolim MKSB. O desenvolvimento motor, a maturação das áreas corticais e a atenção na aprendizagem motora. Rev Digital - Buenos Aires. 2004;10(78). 
Barros KMF T, Fragoso AGC, Oliveira ALB, Oliveira ALB, Cabral Filho E, Castro RM. Do environmental influences alter motor abilities acquisition? A comparison among children from day-care centers and private schools. Arq NeuroPsiquiatr. http://dx.doi.org/10.1590/S0004282X2003000200002

Biscegli TS, Corrêa CE, Romera J, Hernandez JL. Avaliação do estado nutricional e prevalência de carência de ferro em crianças freqüentadoras de uma creche. Rev Paul Pediatr. 2006;24:323-9.

Biscegli TS, Polis LB, Santos LM, Vicentin M. Avaliação do estado nutricional e do desenvolvimento neuropsicomotor em crianças freqüentadoras de creche. Rev Paul Pediatr. 2007;25(4):337-42.

http://dx.doi.org/10.1590/S0103-

05822007000400007

Blauw $\mathrm{CH}$, Hadders M. A systematic review of the effects of early intervention on motor development. Devel Med Child Neurol. 2005;47:421-32.

http://dx.doi.org/10.1017/S0012162205000824

De Souza EAP, Mello BBA. Desenvolvimento infantil - Uma visão comportamental. In: MouraRibeiro MVL, Gonçalves VMG. Neurologia do Desenvolvimento da Criança. 1ed. Rio de Janeiro: Revinter; 2004. Cap.20, p.313-328.

Flehming I. Texto e atlas do desenvolvimento normal e seus desvios no lactente: diagnóstico e tratamento precoce do nascimento até o $18^{\circ}$ mês. São Paulo: Atheneu; 2005.

Habechian FAP, Santos DCC. Atuação fisioterapêutica em ambiente de creche: intervenção em casos especiais. $6^{\mathrm{a}}$ Mostra Acadêmica da UNIMEP. Piracicaba; 2008.

Mansur SS, Neto FR. Desenvolvimento neuropsicomotor de lactentes desnutridos. Rev Bras Fisioter. 2006;10(2):185-91.

Miranda LP, Resegue R, Filgueiras ACM. A criança e 0 adolescente com problemas do desenvolvimento no ambulatório de pediatria. J Pediatr. 2003;79(Suppl 1):S35-S42.

Pilz EML, Schermann LB. Determinantes biológicos e ambientais no desenvolvimento neuropsicomotor em uma amostra de crianças de Canoas/RS. Rev Ciência \& Saúde Coletiva. 2007;12(1):181-90.

http://dx.doi.org/10.1590/S1413-

81232007000100021

Rafael CO, Pires FHB, Fernani DCGL, Mura MLBM. Análise do desenvolvimento motor em crianças com sobrepeso e obesidade através da Escala de Desenvolvimento Motor de Rosa Neto. Rev Temas Sobre Desenvolv. 2010;17(98):69-73.

Santos RS, Araújo APQC, Porto MAS. Diagnóstico precoce de anormalidades no desenvolvimento em prematuros: instrumentos de avaliação. J J Pediatr. 2008;84(4):289-99. http://dx.doi.org/10.1590/S0021-

75572008000400003

Schobert L. O desenvolvimento motor de bebês em creches: um olhar sobre diferentes contextos. Dissertação de Mestrado em Ciências do Movimento Humano. Porto Alegre, 2008.

Souza SC, Leone C, Takano OA, Moratelli HB. Desenvolvimento de pré-escolares na educação infantil em Cuiabá, Mato Grosso, Brasil. Cad Saúde Pública. 2008;24(8):1917-26.

Tecklin JS. Fisioterapia Pediátrica. 3ed. Porto Alegre: Artmed; 2003. p. 42-58.

Willrich A, Azevedo CCF, Fernandes JO. Desenvolvimento motor na infância: influência dos fatores de risco e programas de intervenção. Rev Bras Fisioter. 2006;10(2):185-91. 